\title{
Temperature dependence and single-mode tuning behavior of second-harmonic generation in quantum cascade lasers
}

\section{Citation}

Gmachl, C., N. Owschimikow, A. Belyanin, A. M. Sergent, D. L. Sivco, M. L. Peabody, A. Y. Cho, and F. Capasso. 2004. "Temperature Dependence and Single-Mode Tuning Behavior of SecondHarmonic Generation in Quantum Cascade Lasers." Applied Physics Letters 84 (15): 2751-53. https://doi.org/10.1063/1.1703850.

\section{Permanent link}

http://nrs.harvard.edu/urn-3:HUL.InstRepos:41372510

\section{Terms of Use}

This article was downloaded from Harvard University's DASH repository, and is made available under the terms and conditions applicable to Other Posted Material, as set forth at http:// nrs.harvard.edu/urn-3:HUL.InstRepos:dash.current.terms-of-use\#LAA

\section{Share Your Story}

The Harvard community has made this article openly available.

Please share how this access benefits you. Submit a story.

\section{Accessibility}




\title{
Temperature dependence and single-mode tuning behavior of second-harmonic generation in quantum cascade lasers
}

C. Gmachla) and N. Owschimikow ${ }^{\text {b) }}$

Bell Laboratories, Lucent Technologies, 600 Mountain Avenue, Murray Hill, New Jersey

A. Belyanin ${ }^{\text {) }}$

Department of Physics, Texas A\&M University, College Station, Texas 77843

A. M. Sergent, D. L. Sivco, M. L. Peabody, and A. Y. Cho

Bell Laboratories, Lucent Technologies, 600 Mountain Avenue, Murray Hill, New Jersey

F. Capasso

Division of Engineering and Applied Sciences, Harvard University, Cambridge, Massachusetts 02138

(Received 19 May 2003; accepted 18 February 2004)

\begin{abstract}
Second-harmonic generation (SHG) is reported in quantum cascade (QC) lasers with active regions that also support nonlinear cascades with large second order nonlinear susceptibility. SHG has been measured from 10 up to $250 \mathrm{~K}$ heat sink temperature, with about $1 \mu \mathrm{W}$ of nonlinear power at $10 \mathrm{~K}$ and about $50 \mathrm{nW}$ at $250 \mathrm{~K}$. Single-mode and tunable SHG at $3.5 \mu \mathrm{m}$ wavelength has been measured from single-mode QC distributed feedback lasers operating at the fundamental pump wavelength of $7.0 \mu \mathrm{m}$. Thermal tuning results in a tuning rate for the SHG emission of $\sim 0.2 \mathrm{~nm} / \mathrm{K}$ for temperatures above $\sim 100 \mathrm{~K}$. (c) 2004 American Institute of Physics. [DOI: 10.1063/1.1703850]
\end{abstract}

Intersubband optical transitions in asymmetric coupled quantum wells (QWs) can be designed to exhibit giant resonant optical nonlinearities. ${ }^{1}$ We recently took advantage of this well-known fact for a report of nonlinear light generation - sum frequency and second harmonic generation (SHG) - in quantum cascade (QC) lasers. ${ }^{2}$ As the latter are also based on intersubband transitions in semiconductor QWs, a monolithic approach to the integration of the optical nonlinearity and the fundamental pump QC laser is straightforward and allows for many different configurations. ${ }^{3}$ In a companion paper we report on the optimization of SHG in QC lasers where the optical nonlinearity is collocated with the QWs of the QC-laser active regions. ${ }^{4}$ This earlier work suggests that efficient frequency up-conversion may become an alternative way of generating narrowband, short- to midwave IR radiation $(2-5 \mu \mathrm{m})$ using semiconductor lasers. So far, reports of nonlinear light generation in QC lasers were limited to measurements at cryogenic temperatures $(\sim 10 \mathrm{~K})$ and to Fabry-Pérot type lasers. In light of potential applications in short- to midwave IR sensors, however, high temperature and single-mode operation is required, or at least highly favored.

Therefore, in this letter we report the temperature dependence and single-mode tuning characteristics of SHG in QC lasers. We experimentally demonstrate SHG up to $250 \mathrm{~K}$ heat sink temperature, as well as tunable single-mode emission—simultaneously around 7.0 (fundamental pump) and $3.5 \mu \mathrm{m}$ (SHG) wavelength - in the temperature range from 10 to $250 \mathrm{~K}$. The single-mode tuning rate for the SHG signal is $\sim 0.2 \mathrm{~nm} / \mathrm{K}$ above $\sim 100 \mathrm{~K}$.

\footnotetext{
${ }^{a}$ Present address: Department of Electrical Engineering, Princeton University, Princeton, NJ 08544; electronic mail: cgmachl@princeton.edu

${ }^{b}$ Present address: Technical University of Darmstadt, 64289 Darmstadt, Germany.

${ }^{c}$ Electronic mail: belyanin@jewel.tamu.edu
}

Figure 1 shows a portion of the conduction band diagram, one active region sandwiched between two injector regions, of a QC laser with simultaneous large second-order optical nonlinearity for efficient SHG. Laser action takes place between energy levels 3 and 2. Two second-order nonlinear cascades can be found in the energy level triplets $2-3-4$ and 3-4-5. The existence of two interleaved nonlinear cascades, with $2-3-4$ being the dominant one, is a peculiarity of this particular active region design, and not a

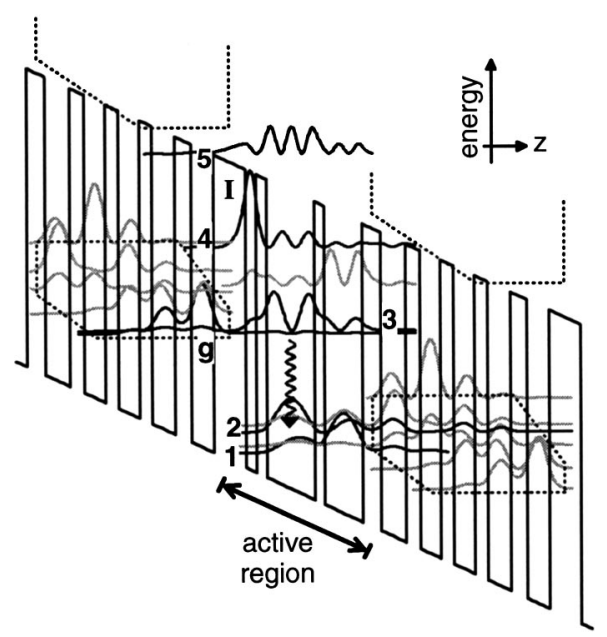

FIG. 1. A portion of the conduction band diagram, one active region sandwiched between two injector regions, and the moduli squared of the essential wavefuntions of wafer D2882. The significant energy levels are labeled 1-5 inside the active region and $\mathbf{g}$ for the ground state of the injector. The dashed lines indicate the extent of the minibands inside the injector regions. The laser transition $(3 \rightarrow 2)$ is marked by a wavy arrow. The thicknesses in nanometers of the QWs and barriers of one period of active region and injector are left to right and starting with the injection barrier " $I$ :" 4.5/1.5/1.5/6.7/1.4/5.3/2.6/3.5/2.0/2.9/1.8/3.0/1.8/3.2/2.3/3.1, the barriers are indicated by bold font, and the underlined layers are doped to 3.0 $\times 10^{17} \mathrm{~cm}^{-3}$. 


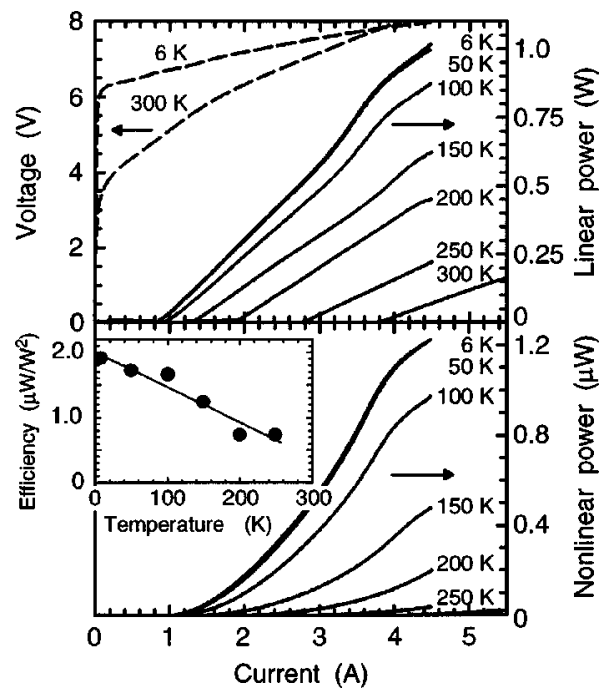

FIG. 2. Linear (top, solid lines) and nonlinear (bottom) light output and voltage (top, dashed lines) vs current characteristics of a $10 \mu \mathrm{m}$ wide and 3 $\mathrm{mm}$ long QC laser of D2886 at various heat sink temperatures as indicated. The inset shows the linear to nonlinear power conversion efficiency as extracted from the $L-I-V$ curves vs the heat sink temperature. The solid line of the inset is a linear least square fit to the data, resulting in a slope of $-5 \mathrm{nW} /\left(\mathrm{W}^{2} \mathrm{~K}\right)$.

general feature of SHG in QC lasers. The QC-laser design shown in Fig. 1 is that of sample D2882, which was used to fabricate single-mode emitting distributed feedback (DFB) devices; an optimized version of this design was realized in wafer D2886 and used for the measurement of the temperature dependence. The quantum design and strength of the optical nonlinearities of both samples, as well as their waveguides and device layout, are in detail described in Ref. 4.

Deep-etched ridge waveguide lasers were processed with stripe widths of $10-16 \mu \mathrm{m}$ and cleaved to a length between 1.5 and $3 \mathrm{~mm}$. The lasers were In-solder bonded to copper heat sinks and attached to the temperature-controlled cold finger of a liquid $\mathrm{He}$ flow cryostat. The lasers were operated with $50-80 \mathrm{~ns}$ long current pulses at $4-84 \mathrm{kHz}$ repetition rate. At such low pulse duty cycles, we can assume that the laser core temperature is very close to the heat-sink temperature.

Figure 2 shows the linear and nonlinear light output and voltage versus current $(L-I-V)$ characteristics of a $10 \mu \mathrm{m}$ wide and $3 \mathrm{~mm}$ long device of D2886 at various heat sink temperatures as indicated. The top panel shows the linear optical power at $\sim 7.5 \mu \mathrm{m}$ wavelength measured with nearly unity collection efficiency from one facet using an uncooled $\mathrm{HgCdTe}$ photovoltaic detector. The lower panel shows the nonlinear SHG light output at $\sim 3.75 \mu \mathrm{m}$ wavelength measured with a cooled InSb photovoltaic detector and collected with $60 \%$ collection efficiency from the same facet. From the linear and quadratic portions of the $L-I$ curves in the top and bottom panel, respectively, an external linear to nonlinear power conversion efficiency can be deduced. The latter is shown as a function of the heat-sink temperature in the inset of Fig. 2.

Figure 3 reports spectra taken of the same device of Fig. 2 at cryogenic temperature as well as at $250 \mathrm{~K}$ heat sink temperature.

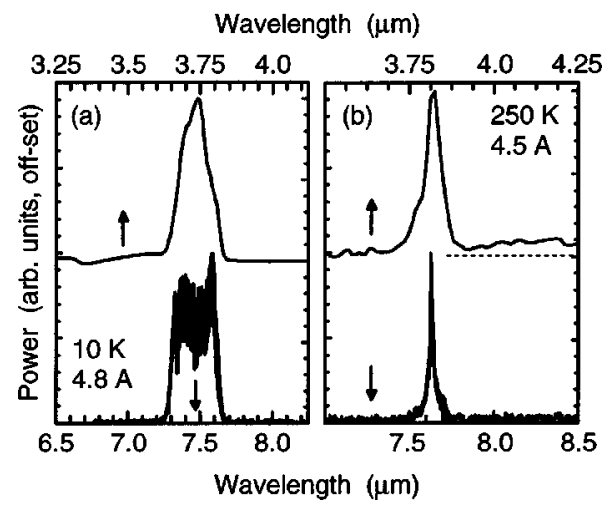

FIG. 3. Emission spectra of QC lasers with integrated second-order nonlinear cascades obtained at $10 \mathrm{~K}$ (a) and $250 \mathrm{~K}$ (b) and at high peak current levels as indicated. The high-resolution spectra in the fundamental wavelength range (bottom traces) have been obtained using a Fourier Transform Infrared spectrometer in rapid-scan mode. The spectra in the short wavelength range (top traces) have been taken in step-scan mode with an InSb detector fitted with a sapphire window and additional quartz/silica glass filter and are low-resolution. The dashed horizontal line in (b) indicates the zero-level for the respective spectrum.

At cryogenic temperatures short wavelength light with power levels up to $\sim 1.2 \mu \mathrm{W}$ has been observed, which attests to an efficient SHG process. As the heat sink temperature is raised, the nonlinear power drops quickly, a combined effect of the reduced power level of the fundamental pump laser and the reduced linear to nonlinear conversion efficiency. Nevertheless, at $250 \mathrm{~K}$, a clear SHG signal can be measured, as shown in Fig. 3(b) top, with a peak power level of $\sim 50 \mathrm{nW}$. Only at $300 \mathrm{~K}$ had the signal disappeared into a rising short-wavelength background. ${ }^{5}$

The reduction of the fundamental optical power with increased heat sink and active region temperature is a wellknown effect with QC lasers (as with most semiconductor lasers), and the laser of Fig. 2, top, is a quite representative example. Nevertheless, QC lasers with higher optical power even at room temperature have been reported, which feature either a significantly larger number of QC-laser active regions in the waveguide core, ${ }^{6}$ were processed as large area or amplifying devices, ${ }^{7}$ or were the result of careful materials engineering. ${ }^{8}$ Therefore, efficient SHG should be possible with QC lasers having peak output power levels in the watt range at room temperature. In addition, the injector regions in sample D2886 were doped about 2.5 times the usual doping level of conventional QC lasers; this helps to increase the nonlinear susceptibility, but also leads to a higher laser threshold and lower slope efficiency and peak optical power at the fundamental frequency.

The deterioration of the external linear to nonlinear power conversion efficiency, conversely, can be understood from a discussion of the second order nonlinear susceptibility $\chi_{2 \omega}^{(2)}$, to which it is closely related through $P(2 \omega)$ $\sim\left|\chi_{2 \omega}^{(2)}\right|^{2} \cdot P(\omega)^{2}$, where $P(\omega)$ and $P(2 \omega)$ are the linear and nonlinear (SHG) power at frequencies $\omega$ and $2 \omega$, respectively. Using the energy level notation of Fig. 1, and focusing on the nonlinear cascade $2-3-4,\left|\chi_{2 \omega}^{(2)}\right|$ is given (somewhat simplified $)^{4}$ by 


$$
\left|\chi_{2 \omega}^{(2)}\right| \propto \frac{e^{3}}{\varepsilon_{0}}\left(N_{3}-N_{2}\right) \frac{z_{23} z_{34} z_{24}}{\left[\left(\hbar \omega-E_{23}\right)^{2}+\left(2 \gamma_{23}\right)^{2}\right]^{1 / 2} \cdot\left[\left(2 \hbar \omega-E_{24}\right)^{2}+\left(2 \gamma_{24}\right)^{2}\right]^{1 / 2}},
$$

where $e, \hbar$, and $\varepsilon_{0}$ are the electron charge, reduced Planck's constant, and vacuum permittivity, respectively. $N_{i}(i=2,3)$ are the electron densities of subband $i$, and $z_{i j}, E_{i j}$, and $2 \gamma_{i j}(i, j=2,3,4)$ the optical dipole matrix elements, energy differences, and transition broadenings between levels $i$ and $j$, respectively. The cascade 3-4-5 also makes a contribution to $\left|\chi_{2 \omega}^{(2)}\right|$, which has a structure similar to Eq. (1). ${ }^{4}$

In Eq. (1), we find several clearly temperature-dependent quantities. In particular, the transition linewidths of QC lasers are known to increase significantly, by $\sim 50 \%$, between 10 and $300 \mathrm{~K}^{6}{ }^{6}$ We estimate values of $2 \gamma_{23}=12 \mathrm{meV}$ and $2 \gamma_{24}=20 \mathrm{meV}$ for $10 \mathrm{~K}$ heat sink temperature and $2 \gamma_{23}$ $=18 \mathrm{meV}$ and $2 \gamma_{24}=30 \mathrm{meV}$ for $300 \mathrm{~K}$. The larger contribution to the drop of $\left|\chi_{2 \omega}^{(2)}\right|$ with increasing temperature thereby results from the increase in $2 \gamma_{24}$, as the increase in $2 \gamma_{23}$ is partly (except for excited state absorption $3 \rightarrow 4$ ) compensated by the increase in $\left(\mathrm{N}_{3}-\mathrm{N}_{2}\right)$. Another source of reduced $\left|\chi_{2 \omega}^{(2)}\right|$ can be found in the thermal shift of the various energy levels that may lead to changed resonance conditions in Eq. (1).

Therefore, to obtain a large second order nonlinear susceptibility also at thermoelectric cooler and up to room temperature, care has to be taken to first achieve narrow line broadening at all temperatures. Highest material quality is one clear avenue to narrow linewidths. Finally, an energy level design that takes into account the thermal shift will also lead to improved high temperature performance.

A large dynamical temperature range is essential for efficient thermal tuning of single-mode QC DFB lasers. In the remainder of the manuscript we discuss the single-mode tuning of SHG of a QC-DFB laser. QC-DFB devices with first order Bragg gratings of $\Lambda_{B}=1.1,1.125$, and $1.15 \mu \mathrm{m}$ period were fabricated from wafer D2882 and operated in conventional fashion. ${ }^{6}$

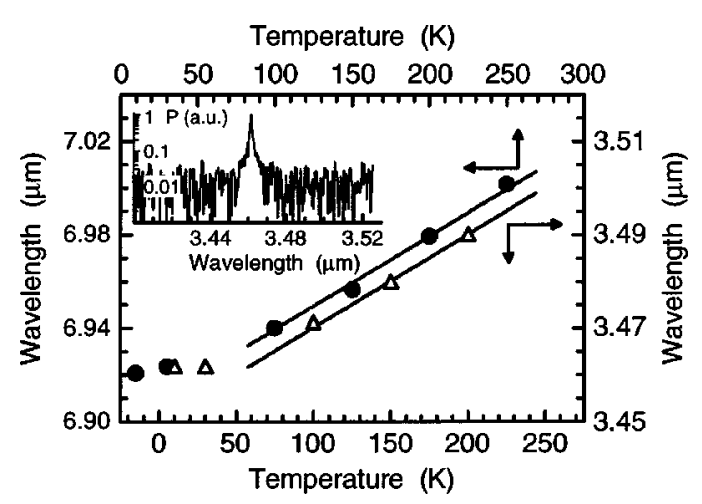

FIG. 4. Single-mode emission wavelength vs temperature for a QC-DFB laser with active regions that also include nonlinear optical cascades. The solid circles indicate the emission wavelength in the fundamental wavelength range and the open triangles in the SHG wavelength range. The solid lines are fitted to the data using slopes of 0.4 and $0.2 \mathrm{~nm} / \mathrm{K}$ for the fundamental and SHG wavelength range, respectively. The inset shows a highresolution spectrum of the single-mode SHG signal obtained in step-scan mode at cryogenic temperatures.
Figure 4 shows the single-mode emission wavelength at both the fundamental pump and SHG wavelengths as a function of the heat sink temperature for a device with $\Lambda_{B}$ $=1.1 \mu \mathrm{m}$. The inset shows a high-resolution shortwavelength spectrum taken at cryogenic temperature. As we obtain single-mode emission for the QC lasers, the SHG signal is also single mode. The fundamental emission wavelength $\lambda_{\omega}$ of the QC-DFB laser is given by $\lambda_{\omega}=2 \cdot n_{\mathrm{eff}}(T)$ $\cdot \Lambda_{B}$, with $n_{\text {eff }}(T)$ being the temperature dependent effective refractive index of the waveguide. The single-mode wavelength $\lambda_{2 \omega}$ of the SHG signal is given by $\lambda_{2 \omega}=\lambda_{\omega} / 2$ $=n_{\text {eff }}(T) \cdot \Lambda_{B}$. Therefore, we expect a SHG tuning rate of half the value of that of the fundamental mode.

In fact, as shown in Fig. 4 we measure tuning rates of $\sim 0.4$ and $\sim 0.2 \mathrm{~nm} / \mathrm{K}$ for the fundamental pump and SHG light, respectively. The latter is very comparable to the tuning rate expected for a QC-DFB laser with fundamental light emission at such short wavelength, ${ }^{6}$ which is not surprising taking into account that the temperature coefficients of the refractive indices of the various materials are only very little wavelength dependent at energies far away from the fundamental energy gap.

The nonlinear optical power measured from the singlemode devices of D2882 was significantly below $1 \mu \mathrm{W}$. Nevertheless, design improvements such as wafer D2886, the data and discussion in Ref. 4, and ongoing work on true phase-matching waveguides indicate that ultimately nonlinear power levels for SHG in the low milliwatt range will be feasible. The limitation to the linear to nonlinear conversion efficiency in the devices presented here was clearly the absence of any phase matching.

The work performed at Bell Laboratories was partly supported by DARPA/US ARO under Contract No. DAAD19-00-C-0096. A.B. acknowledges the support from TAMU TITF Initiative and the Office of Naval Research.

${ }^{1}$ M. M. Fejer, S. J. B. Yoo, R. L. Byer, A. Harwit, and J. S. Harris, Phys. Rev. Lett. 62, 1041 (1989); E. Rosencher, A. Fiore, B. Vinter, V. Berger, Ph. Bois, and J. Nagle, Science 271, 168 (1996); F. Capasso, C. Sirtori, and A. Y. Cho, IEEE J. Quantum Electron. 30, 1313 (1994).

${ }^{2}$ N. Owschimikow, C. Gmachl, A. Belyanin, V. Y. Kocharovsky, D. L. Sivco, R. Colombelli, F. Capasso, and A. Y. Cho, Phys. Rev. Lett. 90, 043902 (2003).

${ }^{3}$ A. A. Belyanin, F. Capasso, V. Kocharovsky, and M. O. Scully, Phys. Rev. A 63, 053803 (2001).

${ }^{4}$ C. Gmachl, A. Belyanin, D. L. Sivco, V. Kocharovsky, M. L. Peabody, N. Owschimikow, A. M. Sergent, F. Capasso, and A. Y. Cho, IEEE J. Quantum Electron. 39, 1345 (2003).

${ }^{5}$ Under high pumping conditions the appearance of a smooth luminescent background is not uncommon in QC lasers. It may be as well thermal in nature as well as result from spontaneous emission by electrons excited into high-lying energy levels.

${ }^{6}$ C. Gmachl, F. Capasso, D. L. Sivco, and A. Y. Cho, Rep. Prog. Phys. 64, 1533 (2001), and references therein.

${ }^{7}$ M. Troccoli, C. Gmachl, F. Capasso, D. L. Sivco, and A. Y. Cho, Appl. Phys. Lett. 80, 4103 (2002)

${ }^{8}$ S. Slivken, A. Evans, J. David, and M. Razeghi, Appl. Phys. Lett. 81, 4321 (2002). 
Applied Physics Letters is copyrighted by the American Institute of Physics (AIP). Redistribution of journal material is subject to the AIP online journal license and/or AIP copyright. For more information, see http:/ojps.aip.org/aplo/aplcr.jsp

Copyright of Applied Physics Letters is the property of American Institute of Physics and its content may not be copied or emailed to multiple sites or posted to a listserv without the copyright holder's express written permission. However, users may print, download, or email articles for individual use. 